\title{
Connective Tissue Disorders: One of The Main Differential Diagnosis of Congenital Hypotonia
}

\section{Keerthana Srinivasan ${ }^{1 *}$ | Rae Infante ${ }^{1}$ | Punita Gupta ${ }^{2}$}

\author{
*Correspondence: Keerthana Srinivasan
}

Address: ${ }^{1}$ Department of Pediatrics, St.Joseph's University Medical Center, 703 Main Street Paterson NJ, 07503, USA;

${ }^{2}$ Department of Pediatric Genetics, St. Joseph's University Medical Center, 11 Getty Ave Fl 2 Paterson NJ, 07503, USA

e-mail $\bowtie$ : keerthusrini21@gmail.com

Received: 26 August 2020; Accepted: 31 August 2020

Copyright: (C) 2020 Srinivasan K. This is an open-access article distributed under the terms of the Creative Commons Attribution License, which permits unrestricted use, distribution, and reproduction in any medium, provided that the original work is properly cited.

\section{ABSTRACT}

Loeys-Dietz syndrome (LDS) is an autosomal dominant connective tissue disorder that has been associated with mutations involving the TGFBR1/2, SMAD2/3, and TGFB2/3 genes. It is characterized by aneurysms, arterial tortuosity, hypertelorism and bifid uvula. We present the case of a full-term infant born with significant hypotonia and congenital anomalies who was eventually diagnosed with LDS. Physical findings were significant for decreased muscle tone, redundant skin on nape of the neck, small chin, widely spaced nipples, and bilateral club feet. Due to the multiple anomalies noted above, genetics consultation was obtained. A chromosomal analysis with reflex to SNP microarray analysis was performed, showing a normal female 46,XX. Given the complex nature of the patient's presentation and normal initial genetics studies, Whole Exome Sequencing (WES) was ordered, revealing a heterozygous -likely De Novo, pathogenic variant in the TGFBFR1 gene (p.Glu239del), consistent with autosomal dominant LDS. Upon literature review, different cases of LDS associated with congenital hypotonia have been described. Nevertheless, medical attention was often sought for other clinical indications such as musculoskeletal and cardiac abnormalities, with some cases mislabeled as Larsen or Beals syndrome. We propose that connective tissue disorders should be included in the main differential diagnosis of congenital hypotonia.

Keywords: Loeys-Dietz Syndrome (LDS), Congenital Hypotonia, Connective Tissue Disorders

\section{Introduction}

Loeys-Dietz syndrome (LDS), an autosomal-dominant connective tissue disorder characterized by vascular findings (arterial aneurysms/dissection), skeletal manifestations, craniofacial features (hypertelorism, bifid/broad uvula and cleft palate) and cutaneous findings (velvety and translucent skin, easy bruising and dystrophic scars) (MacCarrick et al., 2014). It shares many clinical characteristics with Marfan syndrome, with an even higher risk for early aortic dilation and dissection (Meester et al., 2017). Mutations in the TGFBR1 and TGFBR2 genes were the first reported genetic causes of LDS, followed by mutations in the SMAD3 and TGFB2 genes (Lindsay et al., 2012; Loeys et al., 
2005; Regalado et al., 2011). These defects translate in alterations of the transforming growth factor- $\beta$ (TGF- $\beta$ ) signaling, with individuals showing similar cardiovascular, craniofacial, cutaneous, and skeletal features. Although decreased muscle tone has been reported in the neonatal period, medical attention is often turned to musculoskeletal complains, including joint contractures and joint hypermobility (Yetman et al., 2007). We present the case of a full-term infant diagnosed with LDS who was born with congenital hypotonia associated with other anomalies.

\section{Case Presentation}

The infant was born to a 26-year-old mother, G1P0 at 39 weeks of gestation via spontaneous vaginal delivery. Birth weight was $3.845 \mathrm{~kg}$. Pregnancy was achieved naturally. Prenatal history was unremarkable for exposures to medications, alcohol, recreational drugs, tobacco, radiation or maternal illness. A fetal USG showed bilateral choroid plexus cysts, bilateral club feet and bilateral renal cysts. NIPT was positive for Trisomy 13. Amniocentesis was declined. The baby was admitted to the NICU due to respiratory distress and to rule out Trisomy 13. The respiratory distress was likely due to TTN and resolved after 2 days on CPAP. There were no clinical features suggestive of Trisomy 13 but physical exam was significant for redundant skin on nape of the neck, small chin, widely spaced nipples, bilateral club foot, hypotonia, and pectus excavatum. A postnatal renal USG was normal. Head USG revealed small subependymal cysts. Echocardiogram was significant for PFO with left to right flow.

A Genetic consultation was obtained due to the above findings. Initial genetic workup included a chromosomal analysis with reflex to SNP microarray, both of which were normal 46, XX. During a follow up genetics evaluation at two months of age her physical examination was significant for right frontal hair whorl, deep set eyes and epicanthal folds, smooth philtrum, pectus excavatum, long fingers and bilateral clubbed feet in casts, and generalized hypotonia with significant head lag. Given the complex nature of the patient's clinical presentation and the normal karyotype and microarray results, Whole Exome Sequencing (WES) was performed. A heterozygous-likely de novo, pathogenic variant in the TGFBR1 gene was detected, which is consistent with autosomal dominant Loeys Dietz Syndrome.

\section{Discussion}

In 2005, Loeys et al. described a newly recognized genetic syndrome characterized by altered cardiovascular, craniofacial, neurocognitive and skeletal development, now known as Loeys-Dietz Syndrome (Regalado et al., 2011). It is inherited in an autosomal dominant pattern. It is characterized by features such as hypertelorism, bifid uvula, cleft palate, cardiovascular manifestations e.g. vascular aneurysms and dissection, and musculoskeletal features e.g. hypotonia, pectus excavatum or carinatum, scoliosis, joint laxity and arachnodactly (Drera et al., 2009). 
The signs and symptoms of LDS can become apparent anytime from childhood to adulthood, with a variable expressivity and severity. Our patient mainly presented with musculoskeletal features during the newborn period -including hypotonia, pectus excavatum and bilateral club foot, and was diagnosed with Loeys-Dietz Syndrome at five months of age by Whole Exome Sequencing.

Upon literature review, reports of cases with similar musculoskeletal abnormalities during the neonatal period had been made, including diffuse hypotonia, bilateral club foot, and dislocation of hips and knee in addition to cardiovascular findings. These patients were initially misdiagnosed with either Larsen syndrome or Beals syndrome based on their clinical presentation (Caza et al., 2016; Riise et al., 2018). LDS was later diagnosed based on genetic testing. Moreover, more than half of the reported patients required surgical intervention for progressive aortic enlargement and one died due to cardiovascular complications and inadequate cardiac follow-up.

In conclusion, a delay in the diagnosis of a connective tissue disorder like Loeys-Dietz Syndrome during the neonatal period can be life threatening due to the increased risk of aortic aneurysm and aortic dissection. We propose that it is of paramount importance to consider connective tissue disorders as one of the main differential diagnosis of congenital hypotonia in order to decrease morbidity and mortality and improve cardiovascular and developmental outcomes.

Abbreviations: Loeys-Dietz Syndrome (LDS), Transforming Growth Factor-beta Receptor (TGFBR), Single Nucleotide Polymorphism (SNP), Whole Exome Sequencing (WES), Ultrasound (USG), Noninvasive Prenatal Testing (NIPT), Transient Tachypnea of Newborn (TTN), Continuous Positive Airway Pressure (CPAP)

Funding Source: No funding was secured for this study.

Financial Disclosure: The authors have no financial relationships relevant to this article to disclose.

Conflict of Interest: The other authors have no conflicts of interest to disclose.

\section{References}

Caza TN, Mercedes A, Stoppacher R, Catanese CA. A Complex Case of Loeys-Dietz Syndrome: A Case Report and Review of the Literature. Academic forensic pathology 2016; 6: 731-738.

Drera B, Ritelli M, Zoppi N, Wischmeijer A, Gnoli M, Fattori R, Calzavara-Pinton PG, Barlati S, Colombi M. Loeys-Dietz syndrome type I and type II: clinical findings and novel mutations in two Italian patients. Orphanet J Rare Dis 2009; 4: 1-5.

Lindsay ME, Schepers D, Bolar NA, Doyle JJ, Gallo E, Fert-Bober J, Kempers MJ, Fishman EK, Chen Y, Myers L, Bjeda D. Loss-of-function mutations in TGFB2 cause a syndromic presentation of thoracic aortic aneurysm. Nat Genet 2012; 44: 922927. 
Loeys BL, Chen J, Neptune ER, Judge DP, Podowski M, Holm T, Meyers J, Leitch CC, Katsanis N, Sharifi N, Xu FL. A syndrome of altered cardiovascular, craniofacial, neurocognitive and skeletal development caused by mutations in TGFBR1 or TGFBR2. Nat Genet 2005; 37: 275-281.

MacCarrick G, Black JH, Bowdin S, El-Hamamsy I, Frischmeyer-Guerrerio PA, Guerrerio AL, Sponseller PD, Loeys B, Dietz HC. Loeys-Dietz syndrome: a primer for diagnosis and management. Genet Med 2014; 16: 576-587.

Meester JAN, Verstraeten A, Schepers D, Alaerts M, Laer L Van, Loeys BL. Differences in manifestations of Marfan syndrome, Ehlers-Danlos syndrome, and Loeys-Dietz syndrome. Ann Cardiothorac Surg 2017; 6: 582-594.

Regalado ES, Guo DC, Villamizar C, Avidan N, Gilchrist D, McGillivray B, Clarke L, Bernier F, Santos-Cortez RL, Leal SM, Bertoli-Avella AM. Exome sequencing identifies SMAD3 mutations as a cause of familial thoracic aortic aneurysm and dissection with intracranial and other arterial aneurysms. Circ Res 2011; 109: 680-686.

Riise N, Lindberg BR, Kulseth MA, Fredwall SO, Lundby R, Estensen ME, Drolsum L, Merckoll E, Krohg-Sørensen K, Paus B. Clinical diagnosis of Larsen syndrome, Stickler syndrome and Loeys-Dietz syndrome in a 19-year old male: a case report. BMC medical genetics 2018; $19: 1-7$.

Yetman AT, Beroukhim RS, Ivy DD, Manchester D. Importance of the clinical recognition of Loeys-Dietz syndrome in the neonatal period. Pediatrics 2007; 119: e1199-1202. 